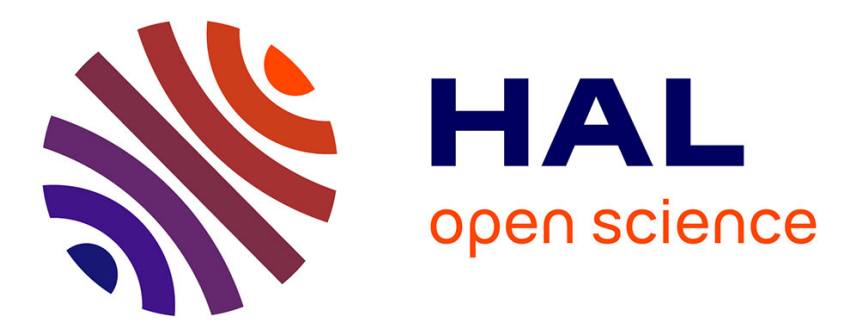

\title{
La difficile prise en charge de l'interface nature-société dans la géographie scolaire française: l'échec de l'introduction du concept de géosystème
}

Christine Vergnolle Mainar, Robert Sourp

\section{- To cite this version:}

Christine Vergnolle Mainar, Robert Sourp. La difficile prise en charge de l'interface nature-société dans la géographie scolaire française: l'échec de l'introduction du concept de géosystème. L'Information géographique, 2006, 70 (3), pp.16-32. hal-02617469

\section{HAL Id: hal-02617469 \\ https://hal-univ-tlse2.archives-ouvertes.fr/hal-02617469}

Submitted on 25 May 2020

HAL is a multi-disciplinary open access archive for the deposit and dissemination of scientific research documents, whether they are published or not. The documents may come from teaching and research institutions in France or abroad, or from public or private research centers.
L'archive ouverte pluridisciplinaire HAL, est destinée au dépôt et à la diffusion de documents scientifiques de niveau recherche, publiés ou non, émanant des établissements d'enseignement et de recherche français ou étrangers, des laboratoires publics ou privés. 


\title{
LA DIFFICILE PRISE EN CHARGE DE L'INTERFACE NATURE-SOCIÉTÉ DANS LA GÉOGRAPHIE SCOLAIRE FRANÇAISE : L'ÉCHEC DE L'INTRODUCTION DU CONCEPT DE GÉOSYSTĖME
}

\author{
Christine Vergnolle Mainar et Robert Sourp
}

Armand Colin | «L'Information géographique »

2006/3 Vol. 70 | pages 16 à 32

ISSN 0020-0093

ISBN 9782200921132

Article disponible en ligne à l'adresse :

https://www.cairn.info/revue-l-information-geographique-2006-3-page-16.htm

Distribution électronique Cairn.info pour Armand Colin.

(C) Armand Colin. Tous droits réservés pour tous pays.

La reproduction ou représentation de cet article, notamment par photocopie, n'est autorisée que dans les limites des conditions générales d'utilisation du site ou, le cas échéant, des conditions générales de la licence souscrite par votre établissement. Toute autre reproduction ou représentation, en tout ou partie, sous quelque forme et de quelque manière que ce soit, est interdite sauf accord préalable et écrit de l'éditeur, en dehors des cas prévus par la législation en vigueur en France. Il est précisé que son stockage dans une base de données est également interdit. 


\title{
La difficile prise en charge de l'interface nature-société dans la géographie scolaire française : l'échec de l'introduction du concept de géosystème
}

\author{
Christine Vergnolle Mainar et Robert Sourp
}

Christine Vergnolle Mainar et Robert Sourp sont maître de conférences

à l'IUFM Midi-Pyrénées et membres du laboratoire Geode UMR 5602 CNRS -

Université de Toulouse II

La rénovation de l'enseignement de la géographie, initiée à la fin des années 1970 en collège et au début des années 1980 en lycée, est une réponse tant à l'évolution des publics scolaires qu'à l'infléchissement des finalités de l'enseignement et au développement de nouvelles approches scientifiques. Elle se traduit par une remise en cause de l'organisation des contenus d'enseignement jusque-là très cloisonnés entre une géographie physique et une géographie humaine héritières de la géographie classique, dans leur contenu comme dans leur hiérarchie. Cet infléchissement de la géographie scolaire se traduit notamment par une plus grande prise en compte de l'homme et des sociétés et par l'affirmation d'une approche plus globale grâce à l'introduction d'une démarche de type systémique.

Dans cette évolution, la question de l'interface entre nature et société et de la façon de l'aborder dans l'enseignement occupe une position centrale. En cela, la géographie scolaire s'inscrit dans un débat plus général qui traverse les champs scientifiques et qui est étroitement lié à l'émergence des préoccupations environnementales. Ainsi dès les années 1970, la recherche en géographie s'est intéressée à l'interaction entre processus naturels et action anthropique en développant notamment le concept de géosystème. Celui-ci ne constitue cependant pas un modèle de référence pour la géographie scolaire en cours de recomposition. Son introduction explicite dans les programmes scolaires est tardive, depuis 1992 seulement, et partielle. En effet, il est peu présent dans les Instructions Officielles et mal articulé avec les autres notions et concepts (milieu, écosystème, paysage...). Il n'a donc eu qu'un faible impact 
sur la structuration du discours de la géographie scolaire et une influence limitée sur les pratiques enseignantes ou la formation des professeurs.

Ce constat nous a interrogé sur les causes de cet échec, sur sa signification quant à la prise en charge par la géographie scolaire de l'interface nature-société, sur ses implications quant à l'évolution de la frontière entre sciences naturelles ou expérimentales et sciences sociales. Au-delà, se pose en profondeur la question du transfert des notions et concepts, des contextes particuliers, des modalités de leur transposition hors de leur domaine scientifique de production.

Pour répondre à cet ensemble de questions, nous avons interrogé la littérature didactique, le texte des programmes successifs et celui des manuels durant la période qui s'étend de l'abandon de la vulgate vidalienne à la mutation actuelle où les contenus d'enseignement sont structurés par des concepts, parmi lesquels apparaît marginalement le géosystème. Nous analyserons les contextes scientifiques et scolaires qui ont prévalu lors des tentatives d'introduction et de ses utilisations ultérieures en envisageant d'un côté l'évolution du concept dans sa sphère d'origine (la recherche en géographie) et d'un autre côté l'évolution de la géographie scolaire dans une perspective large, celle du rapport qu'elle entretient avec les disciplines voisines et qui conditionne son «territoire» d'intervention. Ceci nous permettra de cerner les contraintes et les enjeux qui pèsent sur l'introduction de concepts dans le discours scolaire.

\section{- La prévalence de l'écosystème sur le géosystème (années 1980)}

Le programme de seconde de 1981, ouvre une période de remise en cause du cloisonnement entre géographie physique et géographie humaine et de recherche d'une approche plus globale de l'occupation du milieu par les hommes. Mais bien que le concept de géosystème progresse dans la recherche universitaire, il n'est pas transposé explicitement dans les programmes. C'est au contraire celui d'écosystème qui prévaut, ce qui pose la question des concepts nomades et de leur rôle organisateur dans la sphère qui les accueille.

\section{Le concept de géosystème et sa difficile affirmation dans la recherche en géographie}

Le géosystème permet d'étudier une combinaison dynamique d'éléments biotiques, abiotiques et anthropiques associée à un territoire. Il est l'héritier d'une part de l'approche systémique du milieu naturel conceptualisée par l'écosystème et d'autre part des travaux de la géographie soviétique des années 1960. Formalisé et développé en France par Georges Bertrand, il émerge dans les années 1970 comme un élément de restructuration de la 
recherche en géographie physique et d'ouverture de l'interface entre celle-ci et la géographie humaine.

Le géosystème, dans sa conception initiale (G. Bertrand 1978), « sert à désigner “ un système géographique naturel homogène lié à un territoire”. Il se caractérise par une morphologie, c'est-à-dire par des structures spatiales verticales (les géohorizons) et horizontales (les géofaciès) ; un fonctionnement qui englobe l'ensemble des transformations liées à l'énergie solaire ou gravitationnelle, aux cycles de l'eau, aux biogéocycles, ainsi qu'aux mouvements des masses aériennes et aux processus de géomorphogenèse; un comportement spécifique, c'est-à-dire par les changements d'états qui interviennent dans le géosystème pour une séquence de temps donnée ${ }^{1}$. Le géosystème est donc un outil conceptuel à la fois spatial et temporel. Il permet en effet d'appréhender des superficies étendues et c'est d'ailleurs dans cette perspective qu'il a été utilisé pour la mise en valeur des terres vierges soviétiques. Mais il ouvre aussi la voie à une approche diachronique qui prend en compte à la fois l'évolution des différents processus naturels et celle de l'action anthropique. Il apparaît alors comme un outil permettant d'intégrer les différentes composantes de la géographie physique et de prendre en considération l'interaction société - milieu.

Si le géosystème ainsi défini doit beaucoup à l'écosystème, il en diffère cependant nettement. L'écoystème (P. Blandin et D. Bergandi 2003) est en effet produit par l'écologie scientifique et est un outil d'analyse mis au point dans les années 1930 par Arthur George Tansley et popularisé dans les années 1950 par les frères Odum. Dans son acception première, il permet d'étudier des milieux relativement homogènes et ayant une extension réduite, comme une mare ou une forêt, mais par la suite il est aussi appliqué à des espaces plus vastes et même à la terre dans sa globalité. Dans ces milieux considérés comme des systèmes, ce sont les interactions entre les espèces vivantes et leur biotope ainsi que les flux et les chaînes alimentaires qui deviennent l'objet d'étude principal. Comme l'écosystème, le géosystème repose sur une approche systémique mais il prend en compte l'interaction de facteurs beaucoup plus diversifiés et moins centrés sur la biocénose, allant des formes topographiques au rôle des sociétés. En conséquence, il s'en différencie aussi par les échelles d'espace et de temps sur lesquelles il s'appuie. Il permet notamment de conduire des études à l'échelle régionale, en croisant des analyses localisées avec une approche plus globale. Il offre aussi la possibilité de dépasser le temps cyclique propre au concept d'écosystème et d'intégrer différentes temporalités, celle des processus naturels, celle de l'histoire des sociétés et celle des transformations contemporaines. Il ouvre ainsi la voie à une meilleure intégration du facteur anthropique dans l'approche de l'évolution des milieux.

1. Bertrand C. et G. 2002, Une géographie traversière, l'environnement à travers territoires et temporalités, Arguments, Paris, p. 60. 
De ces deux concepts, c'est celui d'écosystème qui a le plus fort rayonnement. Il obtient en effet une reconnaissance qui lui permet de se "propager » hors de sa sphère d'origine et de mériter le qualificatif de concept «nomade » (I. Stengers, 1987). À l'inverse, le géosystème reste cantonné à son champ disciplinaire où il se heurte d'ailleurs à la résistance d'une partie de la communauté des géographes. Dans le contexte de la restructuration de la géographie scolaire de la décennie 1980, écosystème et géosystème sont donc deux concepts concurrents mais qui ne jouent pas à armes égales.

\section{Le territoire de la géographie scolaire au début des années 1980}

La fin des années 1970 et la décennie 1980 correspondent au début d'une réorganisation de la géographie scolaire, de ses contenus et de ses méthodes d'enseignement, qui remet profondément en cause l'héritage des programmes de 1902.

À l'issue du cycle de réformes qui affecte l'enseignement primaire et les cursus secondaires entre 1880 et 1902 , le champ de connaissances que prennent en charge les différentes disciplines est établi et ne sera pas remis en cause pendant plusieurs décennies. Dans la mosaïque des disciplines de l'enseignement secondaire, la géographie scolaire s'inscrit à la fois dans le champ des sciences de l'homme et de la société et dans celui des sciences naturelles (Fig. 1). La délimitation de ce «territoire» résulte du rapport de forces qui s'est établi avec les sciences naturelles à la suite des vifs débats qui ont opposé géographes et géologues lors de l'élaboration des programmes de $1902^{2}$. Toutefois, dans cette position charnière, le centre de gravité de la géographie est nettement du côté naturaliste et la frontière interne à la géographie, entre géographie physique et géographie humaine, est peu explorée. Dans ce contexte cloisonné, les connaissances enseignées sont organisées dans une logique positiviste et sur un mode encyclopédique, faisant référence à un corpus de notions scientifiques organisées en rubriques closes et hiérarchisées.

Cette organisation connaît un début de remise en cause lorsque les méthodes et les contenus d'enseignement se sont trouvés en décalage par rapport à la demande sociale, lors de la période de massification de l'enseignement secondaire qui a abouti à la création du collège unique en 1975. Toutes les disciplines ont connu une remise en cause de leurs objectifs qui a débouché sur une recomposition interne plus ou moins profonde et durable. La géographie est entrée tardivement dans ce processus, en 1977 pour le collège et en 1981 pour le lycée. Après une longue phase de stabilité, la structure interne de la géographie scolaire connaît alors des bouleversements. La géographie physique est désormais envisagée dans une approche plus systémique et

2. Marchand Ph. 2001, L'histoire et la géographie dans l'enseignement secondaire, textes officiels, tome 1 : 1795 - 1914, INRP, Paris, 782 p. 
globale qui remet en cause la subdivision entre spécialités et l'encyclopédisme. Le cloisonnement entre géographie physique et humaine est également atténué avec un début de prise en compte de la dimension environnementale et des interactions entre l'homme et la nature. Même la hiérarchie entre les deux branches de la géographie commence à être remise en cause.

Dans ce contexte nouveau de prise en compte de l'interface entre nature et sociétés, la question des concepts organisateurs est posée.

\section{L'importation du concept d'écosystème}

Par rapport aux programmes antérieurs, le programme de seconde mis en application à la rentrée 1981 correspond à une rupture fondamentale (confirmée par le programme de 1987) qui intègre la notion de géosystème, mais seulement de façon implicite. Dans les instructions officielles, c'est le concept d'écosystème qui est explicitement mentionné.

Ce programme marque en effet la fin d'une reproduction dans l'enseignement secondaire du cloisonnement des spécialités de la géographie physique, climatologie, hydrologie, géomorphologie, biogéographie et pédologie. Au contraire il introduit une démarche systémique qui vise à mettre en relation les différentes composantes du milieu. Les grands cycles d'échanges sont ainsi abordés de même que la synergie entre les éléments physico-chimiques et les différentes formes de vie. La question de la fragilité des équilibres est aussi traitée dans le cadre de l'analyse des interactions actuelles et passées entre l'action de l'homme et les différents milieux. Ces entrées renouvelées sont traitées principalement à l'échelle de la planète, qui correspond à l'approche traditionnelle des programmes de seconde. Cette démarche s'inspire nettement de celle du géosystème telle qu'elle est développée depuis les années 1970 dans la recherche universitaire française : tentative de refondation d'une géographie physique globale à partir d'une approche systémique, prise en compte de l'action anthropique comme facteur d'évolution des milieux, travail à l'échelle de grands ensembles...

Pourtant c'est le concept d'écosystème, plus ancien, mieux accepté et bien vulgarisé, qui est mentionné dans le programme de 1981, comme dans celui de 1987. Il s'agit là de l'importation dans la géographie scolaire d'un concept élaboré dans un autre contexte disciplinaire. Comme pour tous les concepts «nomades », la question qui se pose est celle de son intégration dans la discipline d'accueil. L'analyse des manuels correspondant à ces deux programmes de géographie générale, montre un mésusage de ce terme qui conduit à un manque de rigueur dans l'analyse géographique. L'écosystème y est le plus souvent défini dans son acception scientifique première à savoir l'étude d'un ensemble très localisé et homogène, mais il est aussi appliqué à l'étude des grands ensembles du globe, notamment les formations végétales et leurs paysages-types, sans toutefois s'inscrire dans une démarche d'écologie du pay- 
Fig. 1 : L'interface nature - société et la frontière entre géographie et sciences de la vie et de la terre

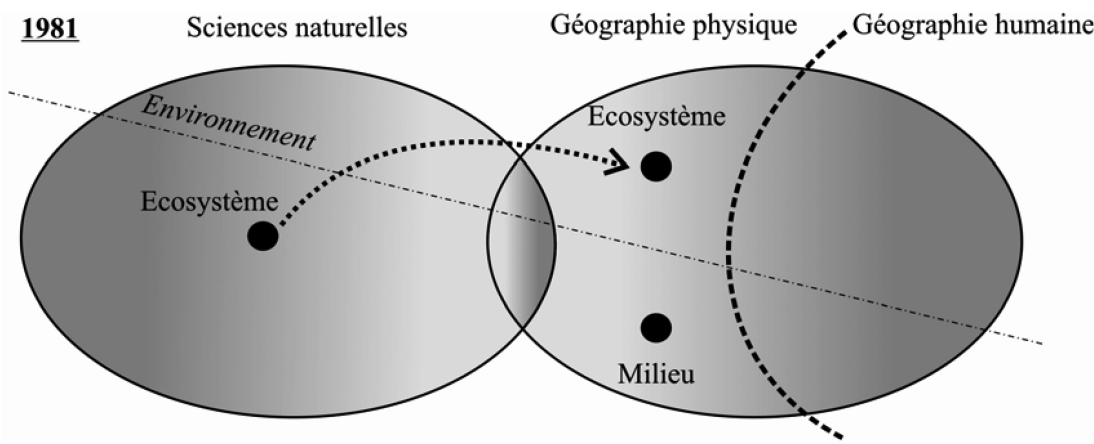

1992 Sciences naturelles Géographie physique , Géographie humaine



$\underline{2001}$ Sciences de la vie et de la terre

Géographie

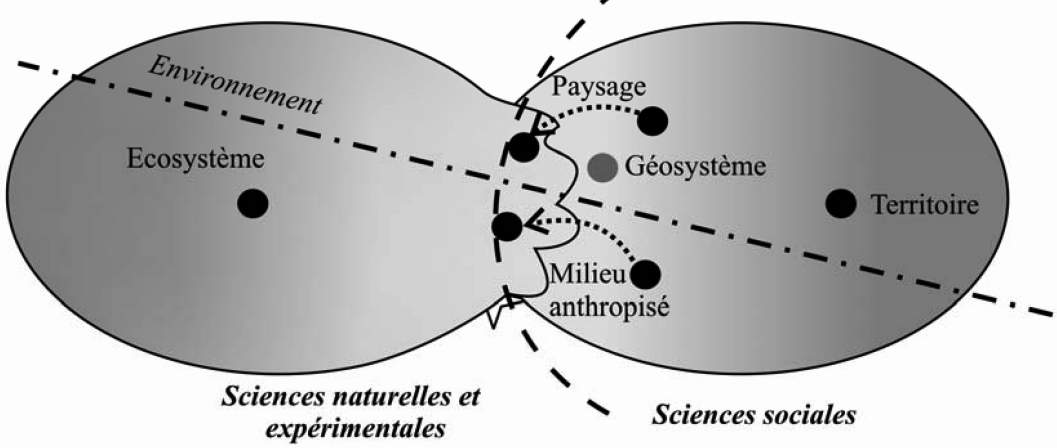

Degré d'ouverture de l'interface

I. entre sciences naturelles et sciences sociales

.$\cdots .2$ Concept nomade $+\longleftrightarrow-$

- $\checkmark$ Degré d'affirmation de l'environnement

Concept émergent

comme objet d'étude transversal

Concept en déclin 
sage au sens propre du terme. Le terme écosystème apparaît plutôt comme équivalent au terme de milieu ou de paysage au sens traditionnel en géographie. Ainsi, quand la géographie a produit des notions qui lui sont propres, l'introduction de l'écosystème ne les remplace pas et conduit à certaines confusions. Au contraire, pour quelques thématiques peu explorées par la géographie classique, comme le milieu marin, des notions associées à l'écosystème, comme la chaîne alimentaire, deviennent un élément structurant.

Dans un contexte d'évolution de la discipline, l'importation d'un concept issu d'un autre champ scientifique a donc en partie débouché sur la persistance des entrées traditionnelles, notamment le milieu, car il n'a pas permis une refondation en profondeur du discours scolaire. Ce concept d'essence naturaliste est en effet décalé par rapport à des contenus d'enseignement qui tendent à se centrer sur l'action de l'homme sur les milieux.

\section{- L'introduction du concept de géosystème dans l'enseignement secondaire (1991-1992)}

Une meilleure connaissance du concept de géosystème par les acteurs de la construction des programmes ainsi qu'une volonté de mieux prendre en charge l'interface nature-société est à l'origine d'une réflexion sur l'intégration de ce concept dans l'enseignement. Cette tentative de transposition intervient à un moment où le concept a déjà connu une évolution : l'idée de territorialisation de la nature, émise par Georges Bertrand dès 1978, est en effet systématisée dans un construit théorique où le géosystème est associé au territoire et au paysage (GTP).

\section{Du géosystème au GTP}

\section{(Géosystème-Territoire-Paysage)}

Georges Bertrand (G. Bertrand, 1991) ${ }^{3}$ partant de l'idée qu'il n'y a «pas de géographie sans paradigme de la nature » intègre la «dimension naturaliste dans le champ des sciences sociales » en proposant d'analyser « non seulement les sociétés dans leurs milieux, mais aussi les milieux dans leurs sociétés ». Cette stratégie d'interface entend étudier les processus d'artificialisation qui produisent «une nature socialisée, territorialisée », c'est-à-dire incluse dans une histoire et dans des processus de patrimonialisation. La méthode d'analyse géographique de la nature peut alors être menée dans «un système conceptuel tridimensionnel » où le géosystème constitue «la dimension anthropique d'un

3. Bertrand G. 1991, La nature en géographie : un paradigme d'interface, GEODOC, Institut de géographie, Université de Toulouse le Mirail, $n^{\circ}$ 34, 16 p. in : Bertrand G. 2002 Bertrand C. et G. 2002, Une géographie traversière, l'environnement à travers territoires et temporalités, Arguments, Paris, (p. 82- 89) 
concept naturaliste ». Le géosystème, en tant que tel, permet de «resituer de façon interactive les éléments qui le composent (la végétation dans le géosystème, le feu dans le géosystème) » mais il est complété par des concepts sociaux, le territoire et le paysage. Le premier pense la dimension naturaliste dans des rapports sociaux, dans des projets de groupes qui véhiculent une analyse des potentialités ou des contraintes naturelles. Ainsi le «naturel des terroirs » n'existe pas mais il y a seulement un projet de récolte, une organisation de la production dont l'effet est de tronquer le géosystème. La réalité du paysage est autre car elle introduit, au-delà de la matérialité qu'exprime le géosystème, les représentations culturelles de la nature que porte en elle la société. Georges Bertrand peut alors écrire ${ }^{4}$ que «le système GTP (...) définit trois champs conceptuels, sémantiques et méthodologiques. Il permet un balayage hiérarchisé et différencié de l'ensemble de l'interface ».

Tel quel, ce cadre théorique n'avait pas pour intention de rester confiné à la recherche mais de permettre, face aux sciences de la nature et au concept intégrateur d'écosystème, d'identifier la géographie grâce à un ensemble de concepts propres. Georges Bertrand pensait alors à l'enseignement en écrivant : «le système GTP pourrait (alors), après quelques ajustements être cet outil didactique » qui lui manque.

\section{Une volonté de transposition du concept de géosystème}

Soucieuses de poursuivre la mutation d'une géographie scolaire encore tributaire du paradigme vidalien et de ses avatars qui la divisait entre une présentation naturaliste préalable à celle des formes de l'activité humaine, les autorités académiques ont largement sélectionné, dans le référent scientifique, tout ce qui allait dans le sens d'un recentrage vers une géographie globale. Dans cette perspective, la théorisation du géosystème et du système GTP avait le double avantage d'offrir une globalisation d'une géographie physique encore éclatée et une focalisation sur les relations d'anthropisation de la nature et de territorialisation. Cette perspective environnementale allait dans le sens recherché et donc a été largement valorisée comme un outil transposable à l'analyse scolaire.

Le colloque d'Amiens en juin 1991 a été un lieu de discussion en direction d'un public d'acteurs du système éducatif où a été proposée cette transposition. L'exposé introductif de Georges Bertrand, centré sur la « didactique de la géographie physique » y posait d'emblée la question du sens de l'enseignement de la géographie physique. Pour lui sa survie n'est possible qu'à condition de considérer la géographie comme une science sociale où la question du rapport des sociétés à la nature est fondamentale, ce qui conduit à rejeter la démarche sectorielle de la géographie physique traditionnelle ainsi que «le modèle réducteur de l'écologie scientifique». L'adoption d'un

4. Bertrand G. 2002, p. 88. 
modèle alternatif propre, le «GTP », s’impose dans le traitement géographique des questions de la nature.

Face à cet apport théorique restait pour la sphère enseignante à résoudre le problème de sa transposition scolaire. Celle-ci n'était possible qu'à condition d'ancrer les contenus d'enseignement en cours de refonte sur des concepts. Le géosystème y paraissait pouvoir être le cadre général qui associe les concepts d'une géographie recentrée, en faisant la synthèse de certains éléments de tradition tels que l'espace ou même le milieu géographique. Dans ce contexte, l'articulation forte sur le paradigme d'interface que supposait le concept de géosystème de manière axiomatique perdait son sens et fut largement ignorée, ce qui eut pour effet de le diluer. Cette déviation est manifeste tant dans les essais de transposition engagés lors du colloque que dans l'aménagement du programme de 1992 et les nouvelles Instructions Officielles de 1996, ainsi que dans les manuels scolaires correspondants. À Amiens, cette transposition du géosystème fut, en effet, tentée en atelier mais son contenu est détourné. Le géosystème y devient un « réseau conceptuel pour penser l'espace géographique » dont le contenu est plus spatial que véritablement sociétal (Fig. 2). La territorialité n'y est inscrite que par sa composante spatiale impliquant l'analyse scalaire et ses corrélats : celle de l'identification des lieux (la localisation et la différenciation) et celle de l'organisation (des structures et du fonctionnement) du système spatial.

\section{Fig. 2 : Géosystème - Concepts-notions pour penser l'espace géographique (atelier 1)}



Source : journées d'études nationales Enseigner la géographie du collège au lycée, juin 1991, Amiens CRDP de Picardie, p. 65.

\section{La transposition avortée}

L'aménagement de programme de 1992 incite à faire comprendre aux élèves « la notion synthétique de milieu ou de géosystème, termes à préférer à celui d'écosystème trop restrictif ». Mais, les Instructions Officielles de 1996 dans leur libellé relatif à la classe de seconde ne font déjà plus référence explicitement à un cadre théorique dont le géosystème est l'élément structurant. Ses 
allusions «aux problématiques de la science géographique actuelle » ne sont pas ou peu explicites quant au nouveau cadre théorique du géosystème. On s'en tient, au mieux à un libellé très traditionnel tel que «les sociétés humaines face aux ressources et contraintes de la nature », «la transformation du milieu par les hommes ». Dans ce cadre, il n'est pas étonnant de voir la transposition du concept tourner court.

Les auteurs de manuels, fortement contraints par des maisons d'édition peu soucieuses de rompre avec une tradition scolaire, accentuent le flou et l'incomplétude de la présentation du géosystème. Ce dernier n'est jamais un fondement paradigmatique de leurs travaux et son statut s'avère ambigu, comme un accompagnement alibi au détour d'un chapitre dont il se veut un approfondissement notionnel. C'est ainsi que l'on peut lire dans le manuel de seconde, pourtant le plus novateur de la génération des ouvrages parus après les Instructions Officielles de 1996, une double page thématique intitulée «l'homme et le géosystème : le milieu montagnard » ${ }^{5}$. À travers un dossier composé de six documents - un paysage enneigé des Alpes suisses, un texte adapté de Georges Viers présentant «la nature alpine » ${ }^{6}$, un schéma simple du phénomène de subduction à l'origine de «la formation d'une chaîne de montagne », un court texte adapté de Paul et Germaine Veyret sur «la vie traditionnelle dans les Alpes $»^{7}$, un plan paysager des pistes de la station de Tignes et un schéma des interactions nature / société à la base de « l'aménagement et la transformation du milieu montagnard» (Fig. 3) - l'exercice proposé aux élèves est « de rechercher les interactions entre les éléments du géosystème montagnard et les sociétés humaines ». Mais la complexité du concept résiste à la présentation et pour le rendre plus conforme aux compétences intellectuelles d'un élève de seconde, il est en fait assimilé au «milieu naturel ». Le concept est certes nommé mais il renvoie continuellement à celui de milieu ${ }^{8}$ en identifiant le couple contraintes / ressources. Dans cette présentation le géosystème perd sa capacité d'outil théorique pour devenir de manière ambiguë un support de l'aménagement et de la transformation des milieux par les hommes.

Cet exemple montre les limites et l'échec des tentatives de transposition scolaire du concept. Pensé pour l'analyse de l'interface société-nature dans le domaine de la recherche universitaire, il devient, dans celui de la sphère d'enseignement, un ensemble composite de concepts réunis pour servir à structurer la continuité et la progressivité des apprentissages dans la discipline, un modèle théorique destiné à faciliter leur pilotage didactique par les enseignants. Deux justifications président à ce dérapage, l'une est dans la lecture que les géographes eux-mêmes ont faite du géosystème proposé par

\footnotetext{
5. Géographie, classe de seconde, Ed. Magnard, 1996, p. $170-171$.

6. Viers G. 1970, Géographie zonale des régions froides, Nathan.

7. Veyret, P. et G. 1979, Les Alpes françaises, Flammarion.

8. Ainsi, les 7 tâches destinées à mobiliser l'activité des élèves sont titrées : « les interactions Homme/milieu ».
} 
Fig. 3 : Le géosystème : exemple de transposition scolaire
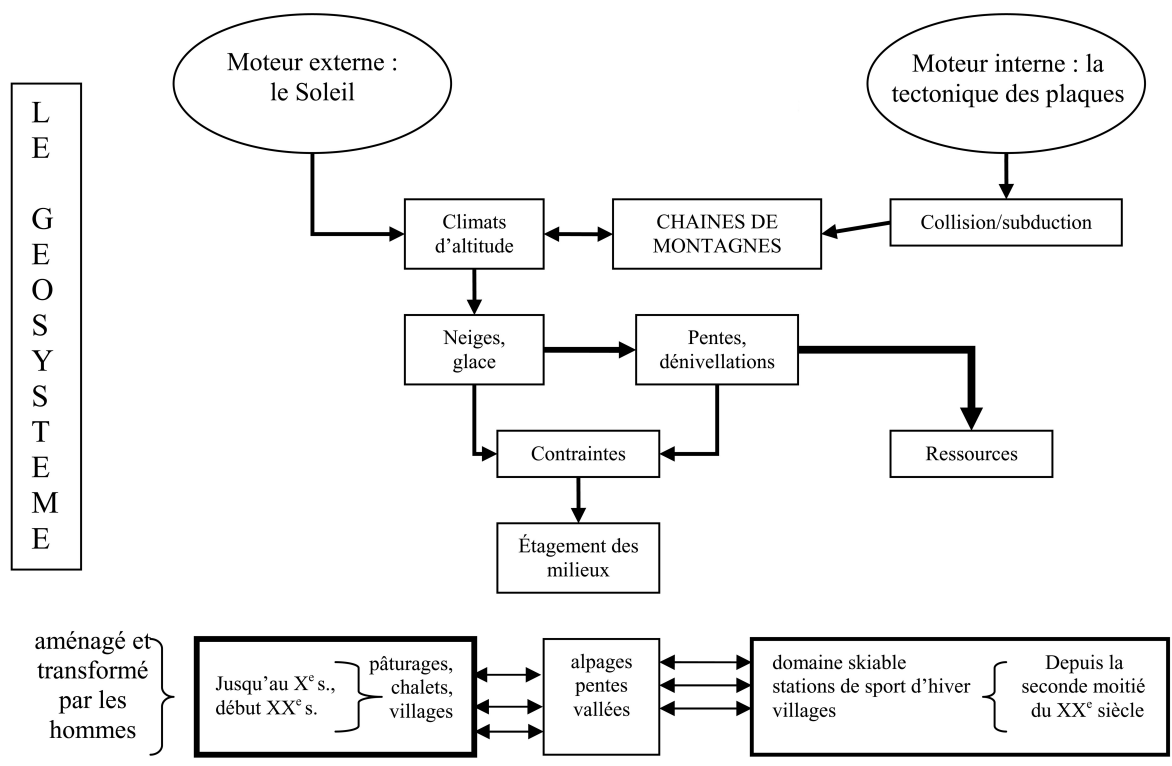

Source : Géographie, classe de seconde, Ed. Magnard, 1996

Bertrand en 1978, l'autre propre au système de la discipline d'enseignement. Dans le géosystème présenté par cet auteur, il y avait une volonté de classement scalaire des phénomènes et de l'étude des interactions entre éléments anthropiques, biotiques et abiotiques, allant du biotope à l'échelle planétaire. Or beaucoup de géographes n'y ont vu qu'un élargissement de la notion de milieu géographique, et donc de milieu humanisé élargi aux dimensions du monde. Dans le domaine de l'enseignement, la nouveauté du concept de géosystème a d'abord séduit par sa capacité à recentrer la géographie, jusque-là éclatée, pour la fonder sur un nouveau paradigme de science du fonctionnement des sociétés dans leurs relations à leur territoire. Mais la persistance de la tradition dans la discipline scolaire n'a fait, au mieux, du géosystème qu'un élément, parmi d'autres, de l'analyse spatiale, sans que soit pensée une théorisation proprement didactique qui en respecte les postulats scientifiques initiaux et en remodèle les contenus.

\section{Le constat actuel : une présence mal définie qui gêne la prise en charge de l'interface nature-société}

Le contexte contemporain est marqué par une évolution (et une diversification) de la prise en charge scientifique de l'interface nature-société et par la 
pénétration des questions environnementales dans les programmes scolaires sous la forme d'objet scolaire transversal. Bien que ces deux tendances résultent d'un mouvement commun, la transposition didactique des avancées scientifiques reste limitée. Le géosystème est toujours mentionné dans les Instructions Officielles et les manuels mais ne joue pas un rôle organisateur, pas plus que les notions et concepts qui, dans la géographie scolaire, lui sont associés. Pourtant dans le contexte contemporain de redéfinition de la frontière entre les disciplines scolaires SVT et géographie, cette lacune est préjudiciable à l'approche de l'interface nature-société.

\section{Évolution des référents scientifiques}

Sous l'impulsion des questions sociales relatives à l'environnement, la recherche universitaire a précisé les outils existants et proposé de nouvelles entrées, multipliant ainsi les concepts susceptibles d'aborder l'interface entre les domaines des sciences sociales et celui de la physique et des sciences de la vie, de la terre et de l'univers (Blandin P. et Bergandi D., 2003).

Georges Bertrand (2002) réaffirme ainsi la nécessité d' enraciner l'environnement dans le territoire et l'histoire longue des sociétés » et inscrit le système Géosystème - Paysage - Territoire dans cette perspective, comme un outil transitoire. "C'est une tentative, d'ordre géographique, pour mâेtriser à la fois la globalité, la diversité et l'interactivité de tout système environnemental. Ce n'est pas une fin en soi. Ce n'est qu'un outil. Ce n'est qu'une étape. Le système GTP ne se substitue à rien. Sa fonction essentielle est de relancer la recherche environnementale sur des bases multidimensionnelles, dans le temps comme dans l'espace, que ce soit dans le cadre de disciplines ou bien dans des formes construites d'interdisciplinarité... ${ }^{9}$. Mais le concept de géosystème cesse d'être au cœur d'une réflexion méthodologique active. Au contraire il se banalise et est largement utilisé dans des applications diverses et parfois déviantes par rapport au concept initial.

Dans le même temps, les recherches menées en histoire de l'environnement, sous l'impulsion des programmes interdisciplinaires du CNRS, ont conduit à la proposition du concept d'anthroposystème (T. Muxart et al. 2003). Ces travaux paléo-environnementaux qui visent à analyser les interactions milieux-sociétés sur la longue durée, ont mis en évidence une co-évolution des systèmes naturels et sociaux. "Formant un système complexe, les systèmes naturel et social s'adaptent et s'ajustent l'un à l'autre de façon permanente ». Pour désigner «cette logique et les objets qui y répondent» Le concept d' «anthroposystème » est proposé. C'est «un système interactif entre deux ensembles constitués par un (ou des) sociosystème(s) et un (ou de)s écosystème(s) naturel(s) et / ou artificia-

9. Bertrand G., op. cit, p. 255. 
lisé(s) s'inscrivant dans un espace géographique donné et évoluant avec le temps $\gg{ }^{10}$. Résolument inscrit dans une approche systémique et revendiquant une filiation avec les concepts d'écosystème et de géosystème, il rencontre certaines critiques car le préfixe anthropo place son centre de gravité du côté des sciences sociales. Pour mieux rendre compte de la prise en charge conjointe par les sciences sociales et naturelles d'autres termes ont été proposés, « socio-écosystème » ou encore « éco-sociosystème ».

Cet aspect sémantique témoigne de la volonté des recherches contemporaines de s'inscrire dans un paradigme d'interface et de prendre comme objet d'étude une nature socialisée. Mais la multiplication des termes et des outils, qui témoigne d'une certaine vitalité de ce champ scientifique, pose problème à une géographie scolaire dont le champ d'intervention se trouve redessiné.

\section{Évolution des frontières et du territoire de la géographie scolaire}

Les disciplines scolaires sont actuellement confrontées à une question forte émanant de la société, celle des contenus à enseigner, qui se décline en deux interrogations : celle de l'utilité sociale des connaissances enseignées et celle de leur adéquation aux savoirs contemporains. Cette tension, qui induit un nouveau découpage des savoirs et de leur prise en charge par les disciplines, concerne particulièrement la géographie (Fig. 1), depuis longtemps en position d'interface entre deux grands domaines de la connaissance.

Les derniers programmes mis en œuvre, ceux de lycée, témoignent de deux facteurs d'évolution dans la géographie scolaire : son ancrage croissant dans les sciences sociales et la prise en compte d'objets d'étude transversaux aux disciplines, comme l'Europe, le patrimoine ou l'environnement. Cette thématique transversale de l'environnement permet de cerner le nouveau partage des rôles avec les disciplines « voisines » (notamment l'histoire, les sciences économiques et sociales ainsi que les sciences de la vie et de la terre) qui conditionne le déplacement du territoire de la géographie et l'établissement de nouvelles frontières. Si, dans le champ de l'environnement, le rapport de forces de la géographie avec l'histoire et les SES a peu évolué, il n'en est pas de même avec les SVT ${ }^{11}$, le partage de territoire établi en 1902 étant profondément remis en cause. En effet, les deux disciplines s'inscrivent en position d'interface entre les deux grands groupes de disciplines enseignées, sciences expérimentales et

10. Muxart T., Vivien F.-D., Villalba B., Burnouf J., 2003 : Des milieux et des hommes, fragments d'histoire croisées, Elsevier, Paris, p. 18.

11. Vergnolle Mainar C. et Desailly B., 2005 : Environnement et sociétés, territoires, risques, développement, éducation, Scéren - CRDP Midi Pyrénées, coll. Focus, Toulouse, $364 \mathrm{p}$. et Vergnolle Mainar C. et Sourp R., 2004 : Les concepts et les modes de raisonnement mobilisés par la géographie scolaire en vue de l'éducation à l'environnement et au développement durable, Journées d'études de didactiques de l'histoire et de la géographie, Caen, 1920 octobre 2004 (actes sur CD ROM) ; Vergnolle Mainar C., 2006 : Quelle convergence entre la géographie et l'histoire dans une perspective d'«éducation à l'environnement pour un développement durable »?, Éducation relative à l'environnement, $n^{\circ} 6$. 
sciences sociales : la géographie n'a certes plus comme objet d'étude le système naturel pour lui-même mais intègre malgré tout les données naturelles dans les facteurs explicatifs qu'elle mobilise; les SVT ont pris en charge l'ensemble des connaissances relatives au système naturel mais en intégrant aussi depuis peu les actions anthropiques. Les recherches poussant à considérer la Nature comme étant socialisée et anthropisée depuis des millénaires, l'intersection entre les deux disciplines scolaires s'avère particulièrement complexe.

Dans ce contexte, la question des concepts organisateurs et du «point de vue » développé par chacune des deux disciplines devient centrale. Concernant la thématique de l'environnement on constate une convergence autour du milieu anthropisé et du paysage, comme objets d'étude et un usage commun de certaines entrées: contraintes, ressources, risques, catastrophes... Ces notions et concepts sont envisagés à travers quatre temporalités : celle du temps géologique, celle de l'histoire des sociétés et de la socialisation de la nature, celle du temps présent et de l'utilisation des ressources naturelles et des valeurs associées à la Nature, celle du devenir, des futurs possibles dans un souci de durabilité des systèmes sociaux et naturels. Ces éléments sont autant de point d'appui pour la construction d'une argumentation qui doit satisfaire une double attente : participer du raisonnement spécifique à la discipline et répondre aux questions dont l'objet d'étude transversal est porteur. En géographie, la construction de ce raisonnement repose sur une mosaïque de concepts, notions et outils. L'écosystème, absent des instructions officielles, est toujours présent dans les manuels dans une acception très semblable à celle en usage depuis le début des années 1980. Le milieu aussi y est très utilisé, souvent pour désigner une combinaison d'éléments naturels. Les trois composantes du GTP connaissent quant à elles des dynamiques contrastées : tant dans les documents officiels que dans les manuels, le paysage reprend de l'importance, le territoire s'affirme et le géosystème occupe une place très discrète sans pour autant disparaître totalement. Par contre, les concepts récemment proposés par la recherche, anthroposystème, socio-écosystème, éco-sociosystème, sont absents.

Dans la géographie scolaire, science sociale en position d'interface avec les sciences expérimentales, la boîte à outils pour traiter de l'interface est donc bien fournie, ce qui pose la question du bon usage de chaque outil.

\section{Mésusage du géosystème}

La présence du terme de géosystème dans les programmes et dans les manuels témoigne d'une volonté d'ancrage du discours scolaire sur une approche conceptuelle universitaire, mais entre l'intention et la réalité, l'écart est grand.

Le géosystème est étroitement associé aux notions d'environnement et de milieu, mais il n'en est pas nettement différencié. Dans le programme de la 
classe de première mis en œuvre en 2003, l'environnement apparaît comme l'équivalent du milieu: "le terme de milieu utilisé traditionnellement en géographie, est devenu pratiquement synonyme d'environnement ». Et dans le programme de seconde (2001), environnement, milieu et géosystème sont peu différenciés : "L'environnement est le milieu physique aménagé. Ses différentes composantes (biosphère, atmosphère, hydrosphère, lithosphère, pédosphère) agissent entre elles et sont en interrelations de nature et d'intensité variées avec les sociétés humaines: l'ensemble forme le géosystème ». Dans les manuels, cette absence de clarification entre les trois termes, milieu - environnement - géosystème, se retrouve pleinement et est même accrue par la persistance de l'usage du concept d'écosystème. Lorsqu'il est mentionné, le géosystème est souvent présenté comme un ensemble spatial d'échelle intermédiaire, produit par l'interaction entre le climat, les eaux, le relief, le sol, le sous-sol (parfois) et les sociétés humaines.

Bien qu'étant une des entrées de ces programmes, le géosystème n'y est que très inégalement présent et est surtout utilisé pour traiter des espaces montagnards. Dans le commentaire du programme de seconde, il est introduit de façon générale dans le point qui traite des «relations que les hommes établissent avec leur environnement », mais il n'est par la suite appliqué qu' aux espaces montagnards. De même rares sont les manuels qui se démarquent de cette approche et associent la notion de géosystème à d'autres thématiques que la montagne (l'eau par exemple). En conséquence, même si les instructions officielles ancrent le géosystème dans une géographie science sociale en stipulant que «les sociétés se trouvent au cœur de géosystèmes plus ou moins anthropisés », ce sont les composantes naturelles du géosystème qui se trouvent valorisées. Dans les chapitres traitant du géosystème montagnard, l'accent est ainsi mis surtout sur les contraintes physiques qui pèsent sur ce système et sur la fragilité des équilibres qui en découlent. Force est alors de constater que le flou qui entoure le terme de géosystème tend à renforcer son ancrage naturaliste et constitue par là un frein à son usage dans des programmes d'enseignement dont les entrées sociales sont au contraire sans cesse réaffirmées.

Dans l'usage effectif du terme géosystème au sein de la géographie scolaire, la confusion entre objet et outil est grande. Dans la recherche universitaire, le géosystème est réaffirmé comme un outil permettant d'analyser un objet d'étude, qui peut être un milieu, un paysage... ${ }^{12}$, alors que dans les programmes et manuels la question du statut du terme est totalement éludée d'où la confusion avec l'environnement et le milieu. Les causes de ce mésusage sont à rechercher dans la résistance de la sphère de l'enseignement et son attachement aux entrées traditionnelles, mais aussi dans l'absence de consensus actuel sur l'approche conceptuelle de l'interface nature-société.

12. Filleron J.-C. (document de travail non publié) 


\section{Conclusion}

L'échec de la transposition du concept de géosystème pose au didacticien et à la communauté des praticiens de la géographie scolaire un problème fondamental : un concept conçu dans un cadre scientifique est-il transposable à la sphère éducative ? Le cas, en tous points comparable, de l'échec de la transposition du concept de «système monde» $(\mathrm{P}$. Clerc, 2002) renforce cette interrogation, sans bien sûr permettre d'en faire une loi. La refondation scolaire des concepts ne va pas de soi car elle interroge tout le système d'enseignement dans lequel elle s'inscrit. Elle ne peut se faire sans qu'en amont une expertise en prépare le champ d'application dans les programmes et qu'en aval la formation des enseignants en facilite la prise en charge. Ce constat nécessite une nouvelle formulation, un nouveau débat et surtout des propositions pour une refondation scolaire de l'usage des concepts.

Dans le champ de l'interface nature-société, cette question est particulièrement importante du fait de l'évolution des approches scientifiques et des enjeux de la thématique environnementale au sein de la société en général et de l'éducation en particulier. Elle ne peut être abordée dans la seule mise en correspondance des géographies universitaire et scolaire, comme y inciterait la tradition didactique. La recomposition en cours dans la recherche en environnement, incite en effet à aborder, dans l'enseignement, les questions environnementales sous forme d'un croisement de points de vue qui respecte les logiques méthodologiques des sciences de la nature et des sciences sociales. Dans ce cadre, la géographie peut avoir sa part en développant une démarche heuristique propre à ces dernières.

\section{$\boldsymbol{\nabla}$ Bibliographie}

Bertrand C. et G., (2002), Une géographie traversière, l'environnement à travers territoires et temporalités, Arguments, Paris, $311 \mathrm{p}$.

Blandin P. et Bergandi D., (2003), "La nature avec ou sans les hommes », La Recherche, hors série $\mathrm{n}^{\circ} 11$, p. 67-71.

Clerc P., (2002), La culture scolaire en géographie : le monde dans la classe, Presses Universitaires de Rennes, Rennes, $185 \mathrm{p}$.

Collectif, (1991), Concepts-notions pour penser l'espace géographique in Journées d'études nationales Enseigner la géographie du collège au lycée, Juin 1991, Amiens, CRDP de Picardie.

Marchand Ph. (2001), L'histoire et la géographie dans l'enseignement secondaire, textes officiels, tome 1 : 1795 - 1914, INRP, Paris, $782 \mathrm{p}$.

Muxart T., Vivien F.-D., Villalba B, Burnouf J., (2003), Des milieux et des hommes, fragments d'histoire croisées, Elsevier, Paris, 214 p.

Vergnolle Mainar C. et Sourp R., (2004), Les concepts et les modes de raisonnement mobilisés par la géographie scolaire en vue de l'éducation à l'environnement et au développement durable, Journées d'études de didactiques de l'histoire et de la géographie, Caen, 1920 octobre 2004 (actes sur CD Rom) 


\section{La difficile prise en charge de l'interface nature-société}

Vergnolle Mainar C. et Desailly B., (2005), Environnement et sociétés, territoires, risques, développement, éducation, Scéren - CRDP Midi Pyrénées, coll. Focus, Toulouse, $364 \mathrm{p}$.

Vergnolle Mainar C., (2006), (sous presse), «Quelle convergence entre la géographie et l'histoire dans une perspective d'"éducation à l'environnement pour un développement durable" ? ", Éducation relative à l'environnement, Québec, Montréal, $n^{\circ} 6$.

Stengers I., 1987 : D'une science à l'autre, des concepts nomades, Seuil, Paris, 387 p. 